\title{
Design of a Data-Driven Controller using Open-Loop Data
}

\author{
Yasuteru Nishiya \\ Graduate School of Advanced Science and Engineering, Hiroshima University \\ 1-4-1, Kagamiyama, Higashihiroshima city, Hiroshima, Japan \\ Takuya Kinoshita \\ Graduate School of Advanced Science and Engineering, Hirosihma University, \\ 1-4-1, Kagamiyama, Higashihiroshima city, Hiroshima, Japan \\ Toru Yamamoto \\ Graduate School of Advanced Science and Engineering, Hirosihma University, \\ 1-4-1, Kagamiyama, Higashihiroshima city, Hiroshima, Japan \\ E-mail: \{nishiya-yasuteru,kinoshita-takuya,yama\}@hiroshima-u.ac.jp \\ http://www.hiroshima-u.ac.jp
}

\begin{abstract}
In recent years, data-driven control has been proposed and extended to a non-linear system by using database. At this time, various data are required to obtain good control performance but the cost is required. In this paper, a new scheme that enables various data generation and control system design from a set of open-loop data is proposed. Besides, the filter is designed to keep the value of the reference signal constant. The effectiveness of the proposed scheme is numerically verified.
\end{abstract}

Keywords: data-driven control, PID controller, response prediction, offline, reference signal, filter

\section{Introduction}

Recently, data-driven control schemes ${ }^{1,2,3}$ have been proposed, and it can calculate control parameters directly from a set of input/output data without system modeling. In addition, a Database-Driven control methods (DD) $)^{4,5}$ have been proposed for nonlinear systems using databases. In a DD control system, various data need to be stored in a database to obtain good control performance, but collecting enough data requires multiple experiments, and it takes time and human cost.

In order to avoid the costs associated with data acquisition, DD control using an Estimated Response Iterative Tuning (DD-ERIT) ${ }^{6}$ has been proposed. It generates estimated input/output data from a closed-loop data set. However, in the ERIT method, a two-degree-offreedom control system must be used to obtain the estimated data. Moreover, the estimation accuracy is degraded when constructing a one-degree-of-freedom control system. Therefore, in this paper, a new control scheme that can generate $\mathrm{I} / \mathrm{O}$ data offline using a set of open-loop data. The proposed scheme is a simple method that requires only the replacement of blocks on the block diagram to generate data. It is also an effective method for the unknown high order systems. Since some prediction I/O data can be obtained offline with experimental data, the time and human costs for experiments can be reduced. Furthermore, the feature of the proposed scheme are as follows:

(i) The frequency response of the system can be estimated.

(ii) It can be extended to control system design.

2. Basic idea of the proposed scheme

(C) The 2021 International Conference on Artificial Life and Robotics (ICAROB2021), January 21 to 24, 2021 


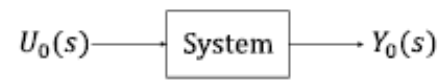

Fig. 1. Block diagram of open-loop system.

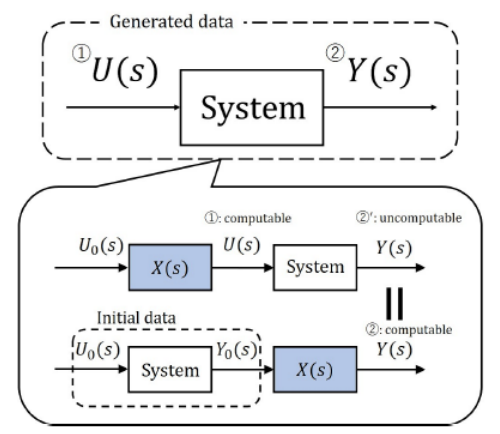

Fig. 2. Outline of the proposed scheme.

In the proposed scheme, open-loop data $U_{0}(s)$ and $Y_{0}(s)$ are obtained in Fig. 1. Next, consider applying an arbitrary input signal $U(s)$ shown in Fig. 3. The experiment is required to obtain $Y(s)$ by applying an arbitrary $U(s)$ when the system is unknown. Therefore, in the proposed scheme, the following $U(s)$ is given by using the initial data $U_{0}(s)$ :

$$
U(s)=X(s) U_{0}(s),
$$

where $X(s)$ denotes the transfer function given by the user to calculate $U(s)$ using $U_{0}(s)$. Here, the output $Y(s)$ in Fig. 2 is equivalent even if the front and back of the blocks are swapped because of the properties of the linear block diagram. Therefore, the output $Y(s)$ is calculated by following equation:

$$
Y(s)=X(s) Y_{0}(s)
$$

From equation (1) and (2), the output $Y(s)$ can be calculated offline by using the initial open-loop data $U_{0}(s), Y_{0}(s)$. Also, in Fig. 2, various input/output data can be generated offline by changing $X(s)$. The following sections describe the control system design using an open-loop data.

\section{Design of a data-driven PID controller using a set of open-loop $\mathrm{I} / \mathrm{O}$ data}

Fig. 3 and Fig. 4 show an overview of the controller design using the proposed scheme. First, a set of data is generated using the proposed scheme according to Fig. 3 and then design the controller $C(s)$ to minimize $\epsilon_{1}(s)$ shown in Fig. 4 based on the generated data. The details

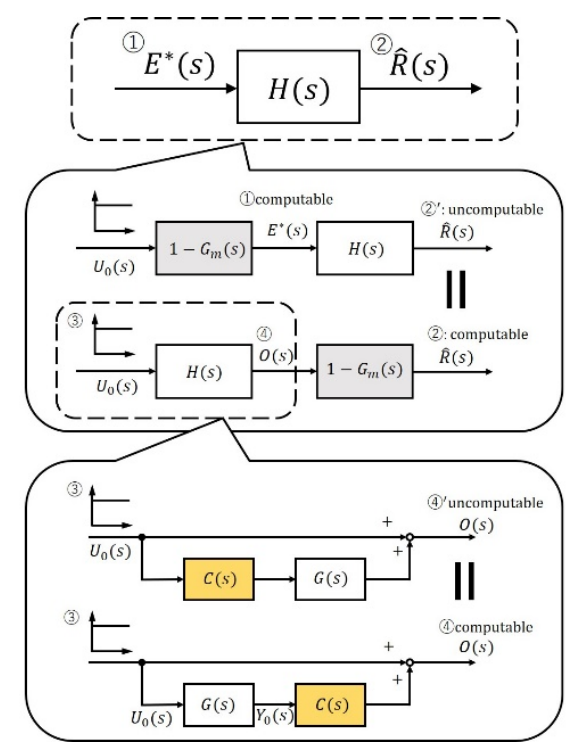

Fig. 3. Generate signal $E^{*}(s)$ and $\hat{R}(s)$ based on the proposed scheme.

of Fig. 3 and Fig. 4 will be described later. First, the output of the reference model $Y_{m}(s)$ is given by

$$
\begin{aligned}
Y_{m}(s) & =G_{m}(s) R(s) \\
& =\frac{G(s) C^{*}(s)}{1+G(s) C^{*}(s)} R(s),
\end{aligned}
$$

where $G_{m}(s)$ denotes the reference model, $G(s)$ denotes the controlled system, $C^{*}(s)$ shows the optimal controller and $R(s)$ represents the reference signal. In this case, the desired error $E^{*}(s)$ is defined by

$$
\begin{aligned}
E^{*}(s) & =R(s)-Y_{m}(s) \\
& =\left(1-G_{m}(s)\right) R(s) .
\end{aligned}
$$

The error $E(s)$ is expressed by

$$
E(s)=\frac{1}{1+G(s) C(s)} R(s) .
$$

From equation (5), $R(s)$ is expressed by

$$
R(s)=(1+G(s) C(s)) E(s) \text {. }
$$

Here, $\hat{R}(s)$ which achieve the desired error $E^{*}(s)$ is introduced by following equation:

$$
\begin{aligned}
\hat{R}(s) & :=(1+G(s) C(s)) E^{*}(s) \\
& =H(s) E^{*}(s) \\
H(s) & :=1+G(s) C(s) .
\end{aligned}
$$

$\hat{R}(s)$ is a reference signal in a closed-loop system, but it is not computable because the parameters of the transfer function $G(s)$ in equation (7) are unknown. Here, $\hat{R}(s)$ is generated based on the proposed scheme, and its overview is shown in Fig. 3. Assuming that the reference signal $R(s)$ and the initial input $U_{0}(s)$ are equivalent step signals, the $R(s)$ in equation (4) can be replaced by 


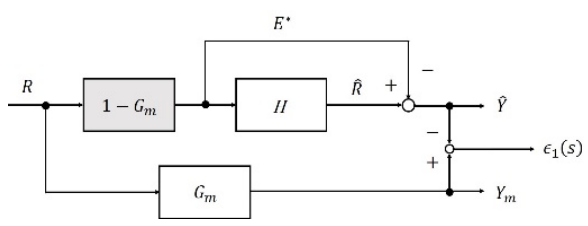

Fig. 4. Block diagram of the minimization problem.

$U_{0}(s) . E^{*}(s)$ in equation (4) can be computed offline because $U_{0}(s)$ has already been obtained as initial input data. However, from equation (7), it is impossible to compute $R(s)$ offline using the desired error $E^{*}(s)$ because the controlled system $G(s)$ is unknown. Therefore, in the proposed scheme, $\hat{R}(s)$ can be computed offline by replacing the order of the blocks, as shown in Fig. 3. Note that, the signal $O(s)$ in Fig. 3 can also be calculated according to the proposed scheme. From the above, $E^{*}(s)$ and $\hat{R}(s)$ can be computed offline for any $C(s)$ using the initial data $U_{0}(s)$ and $Y_{0}(s)$.

However, the original goal of control is to design a controller $C(s)$ in which output $Y(s)$ follows to the reference output $Y_{m}(s)$. The output $\hat{Y}(s)$, where the reference signal is $\hat{R}(s)$ and the error is $E^{*}(s)$ in a closed-loop system, can be expressed by the following equation using (4) and (7):

$$
\widehat{Y}(s)=\hat{R}(s)-E^{*}(s) \text {. }
$$

Fig. 4 shows the relationship between $\hat{Y}(s)$ and $Y_{m}(s)$ using the step signal. At this time, let $Y(s)$ and $Y_{m}(s)$ be Laplace inverse transformed signals $\hat{y}(t)$ and $y_{m}(t)$, respectively, and define the following equation as an evaluation function using the sum of the squares of the errors:

$$
J_{1}=\int\left(y_{m}(t)-\hat{y}(t)\right)^{2} d t
$$

The controller in a closed-loop system is designed by optimizing the control parameters using equation (10). However, the input response is unknown when designing a control system focusing on only the output signal. In this case, the system will be overloaded and cause failure if the input response is excessive. Therefore, the following criterion $J_{2}$ with a weight coefficient $\lambda$ is introduced to consider input signal:

$$
J_{2}=\int\left(\left(y_{m}(t)-\hat{y}(t)\right)^{2}+\lambda(u(t)-u(t-1))^{2}\right) d t,
$$

where $\hat{u}(t)$ is an estimate of the input and the Laplacetransformed value $\widehat{U}(s)$ can be calculated as follow equation:

$$
\widehat{U}(s)=C(s) E^{*}(s) .
$$

\section{Simulation Examples}

\subsection{Controlled system}

The following controlled system is discussed:

$$
G(s)=\frac{s+6}{s^{3}+6 s^{2}+11 s+6} .
$$

The step signal used in the initial experiments $u_{0}(t)$ is equal to 1.0 . The reference output $y_{m}(t)$ is given by

$$
y_{m}(t)=\frac{z^{-1} T(1)}{T\left(z^{-1}\right)} r(t) .
$$

Here, let $T\left(z^{-1}\right)$ be the denominator of the discrete time is defined as follows ${ }^{7}$ :

$$
\begin{gathered}
T\left(z^{-1}\right)=1+t_{1} z^{-1}+t_{2} z^{-2} \\
\left\{\begin{array}{l}
t_{1}=-2 \exp \left(-\frac{\rho}{2 \mu}\right) \cos \left(\frac{\sqrt{4 \mu-1}}{2 \mu} \rho\right) \\
t_{2}=\exp \left(-\frac{\rho}{\mu}\right) \\
\rho=\frac{T_{s}}{\sigma} \\
\mu=0.25(1-\delta)+0.51 \delta
\end{array},\right.
\end{gathered}
$$

where $T_{S}$ denotes sampling time. $\sigma$ and $\delta$ show the parameters related to the rise-time and the damping characteristic, respectively. Here, $T\left(z^{-1}\right)$ was set as follows:

$$
T\left(z^{-1}\right)=1-1.213 z^{-1}+0.368 z^{-2},
$$

where $T\left(z^{-1}\right)$ was designed by setting $\sigma$ as 0.5 and $\delta$ as 0.0 . Here, $T_{S}$ is equal to 0.001 . In addition, the PID controller $C(s)$ is designed as follows:

$$
C(s)=K_{P}+\frac{1}{s} K_{I}+s K_{D}
$$

\subsection{Design of data-driven PID controller}

First, the step response for equation (13) as a set of initial data $u_{0}(t)$ and $y_{0}(t)$ is obtained. Next, Fig. 5 shows the control results of applying the proposed scheme to minimize the evaluation function of equation (10)(9) using the initial data. In Fig. 5, $y(t)$ and $u(t)$ denote the output and input for a closed-loop system with a reference signal $\hat{r}(t) . \hat{y}(t)$ and $\hat{u}(t)$ represent the prediction output and input. Also, $y_{m}(t)$ denote the output of the reference model. In this case, the control parameters were tuned as follows: 

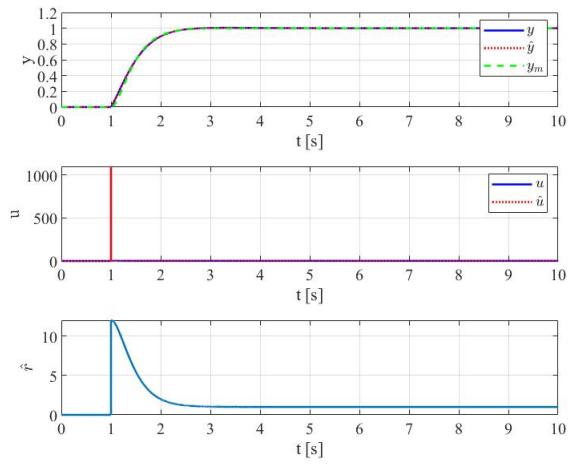

Fig. 5. Control results by the proposed scheme that minimizing equation (10).

$$
K_{P}=0.261, K_{I}=0.167, K_{D}=0.091 .
$$

Fig. 5 shows that the accuracy of $\hat{y}(t)$ and $\hat{u}(t)$ are high. However, the input $u(t)$ changes abruptly and the actuator is heavily loaded since evaluation function in equation (9) is considered only $y(t)$.

Next, the controller is designed by minimizing the evaluation function of equation (11). Fig. 6 shows the control results. In this case, the design the desired characteristic polynomial included in the reference model was set as equation (17), and the weight coefficient $\lambda$ is set as 0.016 . In addition, the control parameters were tuned as follows:

$$
K_{P}=0.223, K_{I}=0.143, K_{D}=1.85 \times 10^{-5} .
$$

Comparing Fig. 6 with Fig. 5, it can be confirmed that the input changes gently and the maximum value becomes smaller by changing the evaluation function. This indicates that the load on the actuator becomes smaller. In addition, Fig. 6 shows the prediction I/O data $\hat{y}(t)$ and $\hat{u}(t)$ are consistent with the actual data $y(t)$ and $u(t)$, respectively. The prediction accuracy is well estimated without degradation.

\section{Conclusion}

In this paper, a new scheme that multiple data can be generated offline from a set of open-loop I/O data has been proposed. According to the proposed scheme, controller can be designed by generating data offline from a set of open-loop I/O data. The effectiveness of the proposed scheme has been verified by a numerical simulation. It is possible to design the controller to avoid overloads in actual machines by setting the evaluation functions appropriately. The future work for this study is
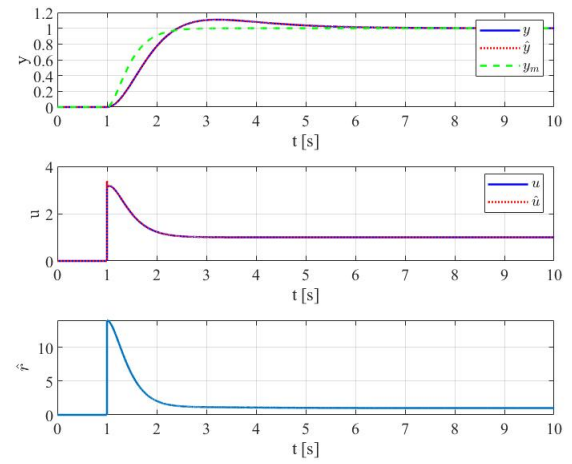

Fig. 6. Control results by the proposed scheme that minimizing equation (11).

planned to design a controller for nonlinear systems and verify the effectiveness of the proposed scheme by experiment.

\section{References}

1. S. Soma, O. Kaneko and T.Fujii, "A New Approach to Parameter Tuning of Controllers by Using One-Shot Experimental Data: A Proposal of Fictitious Reference Iterative Tuning (in Japanese)", Trans. of the Institute of systems, Control and Information Engineers, 17(2) (2004) pp. 528-536.

2. M.C. Campi, A. Lecchini and S.M. Savaresi, "Virtual Reference Feedback Tuning: A Direct Method for the Design of Feedback Controllers", Automatica, 38(8) 2002, pp. 1337-1346.

3. T. Nakamura, O. Kaneko, "Estimated Response Iterative Tuning of Feed-Forward Controller in the Two-Degree of Freedom Control System (in Japanese)", The society of Instrument and Control Engineers, 2017

4. T. Yamamoto, K. Takao and T. Yamada, "Design of a Data-Driven PID Controller", IEEE transactions on control systems technology, 17(1), 2009, pp. 23-29.

5. S. Wakitani, T. Yamamoto and B. Gopaluni, "Design and Application of a Database-Driven PID Controller with Data-Driven Updating Algorithm”, Industrial \& Engineering Chemistry Research, 58(26), 2019, pp. 11419-11429.

6. R. Okada, T, Kinoshita and T. Yamamoto, "Design of a Database-Driven Controller Using Estimated Data", Proceedings of the Society of Instrument and Control Engineers Annual Conference 2019, WeB02.3, Hiroshima.

7. T. Yamamoto, S. L. Shah, "Design and Experimental Evaluation of a Multivariable Self-Tuning PID Controller", IEEE Proc. of Control Theory and Applications, 151, 2004, pp. 645-652. 